\title{
Hanle effect and electron spin polarization in InAs/GaAs quantum dots up to room temperature
}

Jan Beyer, Irina Buyanova, S Suraprapapich, C. W. Tu and Weimin Chen

\section{Linköping University Post Print}

N.B.: When citing this work, cite the original article.

Original Publication:

Jan Beyer, Irina Buyanova, S Suraprapapich, C. W. Tu and Weimin Chen, Hanle effect and electron spin polarization in InAs/GaAs quantum dots up to room temperature, 2012, Nanotechnology, (23), 13, 135705.

http://dx.doi.org/10.1088/0957-4484/23/13/135705

Copyright: Institute of Physics http://www.iop.org/

Postprint available at: Linköping University Electronic Press

http://urn.kb.se/resolve?urn=urn:nbn:se:liu:diva-75096 


\title{
Hanle effect and electron spin polarization in InAs/GaAs quantum dots up to room temperature
}

\author{
J Beyer ${ }^{1, *}$ I A Buyanova ${ }^{1}$, S Suraprapapich $^{2}$, C W Tu ${ }^{2}$ and W M Chen ${ }^{1, *}$ \\ ${ }^{1}$ Department of Physics, Chemistry and Biology, Linköping University, 58183 \\ Linköping, Sweden \\ ${ }^{2}$ Department of Electrical and Computer Engineering, University of California, La \\ Jolla, CA 92093, USA \\ E-mail: beyer@ifm.liu.se,wmc@ifm.liu.se
}

\begin{abstract}
The Hanle effect in InAs/GaAs quantum dots (QDs) is studied under optical orientation as a function of temperature over the range of 150-300 K, with the aim to understand the physical mechanism responsible for the observed sharp increase of electron spin polarization with increasing temperature. The deduced spin lifetime $T_{s}$ of positive trions in the QDs is found to be independent of temperature, and is also insensitive to excitation energy and density. It is argued that the measured $T_{s}$ is mainly determined by the longitudinal spin flip time $\left(T_{1}\right)$ and the spin dephasing time $\left(T_{2}{ }^{*}\right)$ of the studied QD ensemble, of which both are temperature-independent over the studied temperature range and the latter makes a larger contribution. The observed sharply rising QD spin polarization degree with increasing temperature, on the other hand, is shown to be induced by an increase in spin injection efficiency from the barrier/wetting layer and also by a moderate increase in spin detection efficiency of the QD.
\end{abstract}

PACS numbers: 73.21.La, 78.67.Hc, 72.25.Fe

\section{Introduction}

Spins in semiconductor quantum dots (QDs) are the subject of extensive current research due to their weak interaction with the solid state environment, resulting in long spin lifetimes and the proposal for their application in spintronics and quantum information technology [1-3]. To this end, studies of spin injection and detection are important tasks to advance both our understanding of the relevant physical processes and to guide the design of improved structures for spintronic applications. Spin-related properties of QDs have in past years been investigated extensively, mainly at low temperatures, largely thanks to the availability of modern single-dot optical spectroscopy such as micro-photoluminescence ( $\mu$-PL) and scanning near-field optical microscopy (SNOM) [4-10]. Unfortunately, applications of these techniques for studies of QDs have been extremely restricted at elevated temperatures due to drastically decreasing emission intensity with increasing temperature. As a result, detailed studies and understanding of spin properties of QDs at temperatures close to room temperature (RT), highly relevant for practical applications, remain very limited.

Recently, we reported a dramatic increase in electron spin polarization degree of InAs/GaAs QDs at high temperatures [11]. Polarization values up to $35 \%$ in the QD ground state at RT have been found, indicating at least equally high spin detection efficiency. In this work we carry out detailed studies of the Hanle effect [12], i.e. the depolarization effect in a transverse magnetic field, in InAs/GaAs QDs. Our aim is to determine the temperature dependence of the QD electron spin lifetime 
over the temperature range close to RT and its implication on the physical mechanism responsible for the observed rising of electron spin polarization with increasing temperature and the resulting high spin polarization at RT.

Previous studies of the Hanle effect in QDs were conducted at lower temperatures [13-17] and were mainly concentrated on characterization of negatively charged QDs. There, a dominant low-field contribution to spin depolarization curves was observed and was argued to arise from the resident electron left behind after recombination of negative trions. From low-temperature Hanle measurements of single QDs [13], there is evidence that the half-width of Hanle curves is significantly larger for positive trions than for negative trions, indicating a shorter spin lifetime for the former. However, detailed studies of the Hanle effect in positively charged QD ensembles remain few so far and are still lacking at temperatures close to RT.

\section{Samples}

We studied a set of self-assembled InAs based QD structures grown by molecular beam epitaxy (MBE) on (001) oriented GaAs substrates. Differing growth conditions between the samples resulted in the growth of structures consisting of either single QDs (SQD sample), laterally aligned double QDs (DQD sample) or lateral rings of five to seven QDs (QDR sample) residing on a thin InAs wetting layer (WL). Details on the growth process can be found in Refs. [18] and [19]. Due to residual p-type doping during the MBE growth, photoluminescence (PL) signals from the ground state of the ensemble QDs are dominated by recombination from positively charged excitons (called positive trions) over the studied temperature range of $150-300 \mathrm{~K}$. We used a tuneable Ti:sapphire laser as an excitation source. A linear polarizer in conjunction with a broadband quarter-wave plate placed along the path of the excitation beam allowed control of the excitation beam polarization state to achieve circularly polarized excitation. The samples were held in a cryostat equipped with a superconducting split-coil magnet and the direction of the magnetic field was oriented along the sample surface. Both optical excitation and detection were carried out along the direction perpendicular to the sample surface in a backscattering geometry. PL polarization was resolved by the use of either a rotatable broadband quarter-wave plate or a photoelastic modulator (PEM), together with a linear polarizer. Spectrally resolved PL detection was achieved by a grating monochromator equipped with either a liquid nitrogen cooled CCD camera or a liquid nitrogen cooled Ge-detector.

\section{Results and discussion}

For optical orientation, the photon energy of the circularly polarized excitation light was tuned to generate spin-polarized carriers in the GaAs barriers and the WL that were subsequently injected into the QDs. In addition, quasi-resonant excitation of the QDs was also carried out with an excitation photon energy below the WL bandgap. Circularly polarized optical excitation in the GaAs barrier allows creation of spin oriented electron-hole pairs due to the well-known selection rules and a difference in oscillator strength between the optical transitions from the heavy-hole (hh) valence band (VB) to the conduction band (CB) as compared to the transitions from the light-hole (lh) VB to the CB [12]. In the WL, both compressive strain and quantum confinement split the hh and $\mathrm{lh} V \mathrm{VB}$, which improves spin creation efficiency when exciting only free carrier transitions from the hh VB to the $C B$, further on denoted by hh-e transitions. As holes typically experience strong spin relaxation in higherdimensional structures, such as the two-dimensional WL or the three-dimensional GaAs barriers, they may be regarded as unpolarized when reaching the QD ground state. The electrons, however, retain their spin polarization to a certain degree during capture and relaxation into the QD ground state. The maximum electron spin polarization degrees, defined by $\rho=\left(n_{\uparrow}-n_{\downarrow}\right) /\left(n_{\uparrow}+n_{\downarrow}\right)$, generated by optical orientation in the GaAs barrier and the WL (via only hh-e) are $-50 \%(+50 \%)$ and $-100 \%(+100 \%)$ under $\sigma^{+}\left(\sigma^{-}\right)$excitation, respectively. ( $n_{\uparrow}$ and $n_{\downarrow}$ denote concentrations of spin-up and spin-down electrons, respectively.) If the ground state of the QDs is already occupied by a resident hh, then the incoming electron-hole-pair will form a positively charged trion $\mathrm{X}^{+}$, where the remaining electron spin polarization $\rho$ determines the circular polarization degree of the luminescence $P=-\rho$ due to the validity of the same optical selection rules in the QD as in the WL. Here, $P=\left(I^{\sigma+}-I^{\sigma-}\right) /\left(I^{\sigma+}+I^{\sigma-}\right)$ where $I^{\sigma+}$ and $I^{\sigma-}$ denote the intensities of $\sigma^{+}$and $\sigma^{-}$polarized PL, respectively. 
In Hanle measurements, optically oriented electron spins start to precess around the field direction of a transverse magnetic field $B_{\mathrm{x}}$, with a field-dependent Larmor frequency $\Omega=\mu_{\mathrm{B}} g B_{\mathrm{x}} / \hbar$. Here $\mu_{\mathrm{B}}$ is the Bohr magneton, $g$ the transverse electron $\mathrm{g}$-factor and $\hbar$ the reduced Planck constant. The spin precession, leading to a reduction in spin polarization degree along the optically oriented spin axis (i.e. $z$-axis), will be terminated by events of spin relaxation (characterized by a time constant $\tau_{\mathrm{s}}$ ) and decay (characterized by a lifetime $\tau$ ). Here $\tau_{s}=\left(1 / T_{1}+1 / T_{2}^{*}\right)^{-1} . T_{1}$ and $T_{2}^{*}$ represent spin-flip and spin dephasing time, respectively. In an inhomogeneous ensemble, the ensemble spin relaxation time $\tau_{\mathrm{s}}$ is often found to be dominated by the spin dephasing time $T_{2}^{*}$ of the ensemble where individual spin dephasing (described by $T_{2}$ ) plays a negligible role. With increasing transverse magnetic field, the Larmor frequency increases and thus the spin precession accelerates, thereby depolarizing the observed luminescence. The resulting Hanle curve, i.e. a plot of polarization degree $P$ as a function of $B_{\mathrm{x}}$, follows a Lorentzian lineshape according to [12]

$$
P\left(B_{\mathrm{x}}\right)=P(0) \frac{1}{1+\Omega^{2} T_{\mathrm{s}}^{2}}=P(0) \frac{1}{1+B_{\mathrm{x}}^{2} / B_{1 / 2}^{2}}
$$

where $P(0)$ is the polarization degree in zero magnetic field and $T_{\mathrm{s}}$ the spin lifetime of the electrons. As discussed above, $T_{\mathrm{s}}$ is determined by both $\tau$ and $\tau_{\mathrm{s}}$ through the relation $1 / T_{\mathrm{s}}=1 / \tau+1 / \tau_{s}$. From the half-width at half maximum $B_{1 / 2}=\hbar / g \mu_{\mathrm{B}} T_{\mathrm{s}}$, the scaled spin lifetime $g T_{\mathrm{s}}$ can be readily obtained. Below we will use this scaled spin lifetime to compare relative changes of spin lifetime for each sample at different temperatures and excitation energy/density, assuming no drastic change in $g$ over the studied temperature range.

In figure 1 we show Hanle curves of the SQD sample at a temperature of $250 \mathrm{~K}$ taken under $\sigma^{+}$ excitation above the energies of the GaAs barrier and the hh-e transitions in the WL, and also at an energy below the WL bandgap (i.e. quasi-resonant (qr) excitation). The excitation power was carefully chosen under each excitation condition such that the QD PL intensity, i.e. the density of the monitored trions, remains nearly the same to avoid any possible complications arising from different degrees of state-filling and carrier interactions incurred under excitation with different photon energies. As can be clearly seen, an identical $B_{1 / 2}$ was obtained independent of the excitation energies, from which a scaled spin lifetime of $g T_{\mathrm{s}} \approx 13 \mathrm{ps}$ can be deduced. This finding provides compelling evidence that the observed Hanle effect is dominated by the Larmor precession of carrier spins in the QDs, with negligible contributions from the GaAs barrier and WL. The responsible carriers cannot be the holes in the positive trions as the spin-singlet of the two paired holes in the trion ground state should not be affected by the magnetic field. The spin dephasing time of the residual hole in the QD ground state after the trion recombination was shown to be rather long (14 ns) [20], which can also be excluded as being responsible for the observed short spin lifetime. Hence, it must be the spin precession of the trion electron that has led to the observed Hanle curves. In view of a typical electron g-value of 0.5 reported in the literature for similar InAs QDs [21], the effective spin lifetime of the QD trion electron can be deduced as $T_{\mathrm{s}} \approx 26 \mathrm{ps}$. The determined value seems to be in agreement with earlier studies of InAs QDs at temperatures approaching the lowest limit of our temperature range at around $100 \mathrm{~K}$ [14]. 


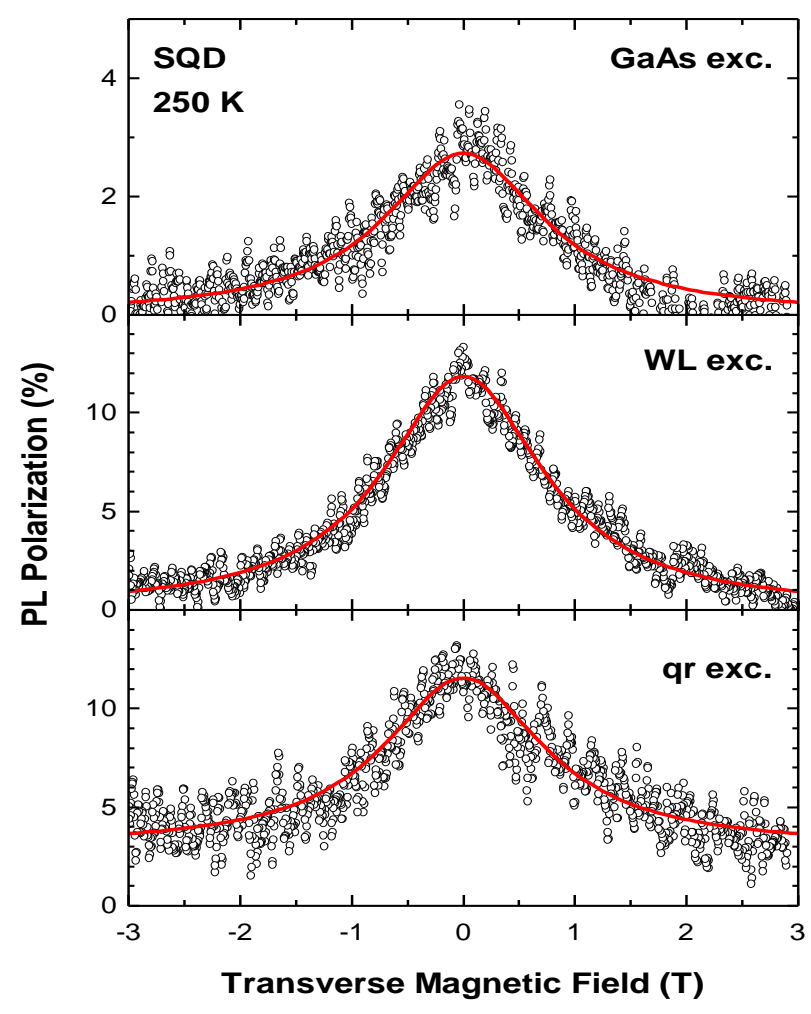

Figure 1. Hanle curves for the SQD sample at $T=250 \mathrm{~K}$, taken under optical orientation with photon energies above the bandgaps of the GaAs barrier (the top curve) and the WL (the center curve) and under the quasi-resonant condition (the bottom curve). The symbols are the experimental data, and the solid lines are the Lorentzian curve fittings based on (1) with the fitting parameter $g T_{\mathrm{s}}=13 \mathrm{ps}$. The excitation power was chosen for each excitation condition such that a similar QD PL intensity was maintained.

To reveal the temperature dependence of the spin lifetime, we performed Hanle measurements over a wide range of temperature between 160-290 K. The results are displayed in figure 2, showing that the width of the Hanle curves is insensitive to measurement temperature in all of the studied QD samples. This finding must mean that $g T_{\mathrm{s}}$ remains the same over the entire temperature range covered in our studies.

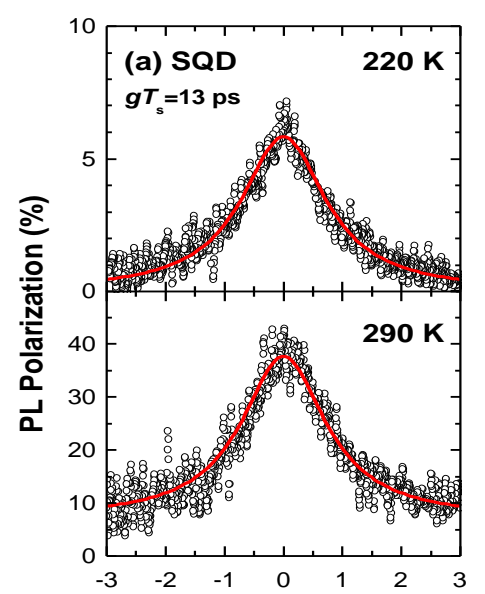

Transverse Magnetic Field (T)

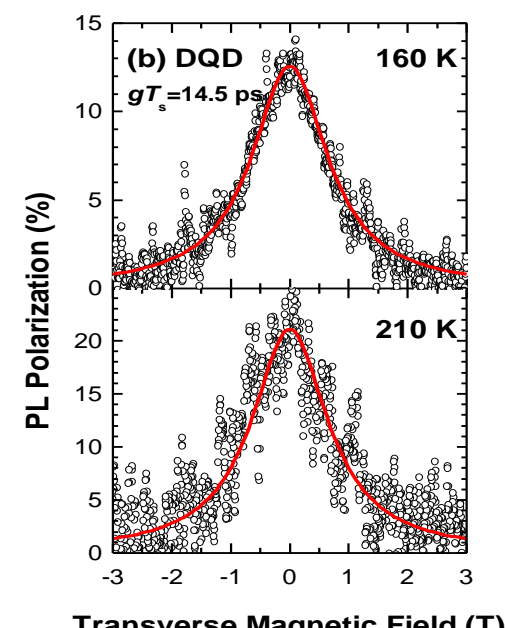

Transverse Magnetic Field (T)

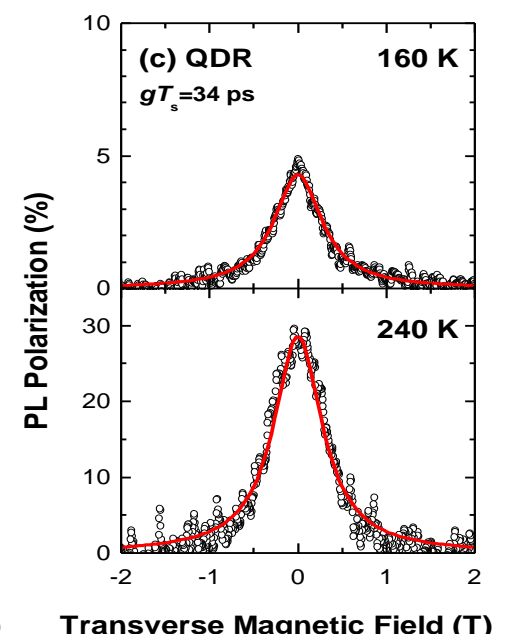

Transverse Magnetic Field (T)

Figure 2. Hanle curves at different temperatures for the SQD (a), DQD (b) and QDR (c) sample. The symbols are the experimental data, and the solid lines are the Lorentzian curve fittings based on (1) with the specified values of the fitting parameter $g T_{\mathrm{s}}$. 
Let us now analyze the relative importance of the three contributions to the trion electron spin lifetime, i.e. $1 / T_{\mathrm{s}}=1 / \tau+1 / T_{1}+1 / T_{2}^{*}$. First of all, the contribution from the trion decay, $\tau$, should be negligible for the following reason. If we assume that its contribution is significant, $\tau$ should then be in the same order of magnitude as $T_{s}$, i.e. tens of ps. This value is much shorter than the radiative lifetime of the trions, which is typically in the order of $1 \mathrm{~ns}$ [22-24]. This large difference must mean that $\tau$ is determined by non-radiative decay processes of the trions, most likely due to thermal activation to exited states or carrier escape from the QDs to the WL. Such non-radiative decay processes are known to be thermally activated, and therefore should be strongly (i.e. exponentially) temperature dependent. This contradicts the experimental fact that $T_{S}$ is insensitive to temperature, which consequently rules out the possibility of a significant contribution of the trion decay in the spin lifetime determined from the Hanle curves. $\tau$ is thus required to be much larger than $T_{S}$, such that a thermal variation of the former will not significantly affect the latter.

Unfortunately, independent measurements of the other two time constants were not possible in our case due to the weak PL close to RT that is beyond the spectral and sensitivity range of the transient polarized PL spectroscopy available to us. Therefore the following discussion will largely be based on values reported in the literature. The spin relaxation time $T_{1}$ was shown in earlier studies to exhibit only very weak temperature dependence in p-doped QDs, decreasing from $\sim 78$ ps at $200 \mathrm{~K}$ to $\sim 72 \mathrm{ps}$ at $300 \mathrm{~K}$ [25]. This value is markedly larger than the measured $T_{\mathrm{s}} \approx 26 \mathrm{ps}$, which indicates that it might not be the sole contribution to $T_{s}$. A sizable contribution from $T_{2}^{*}$ is thus implied. Assuming an approximate average value of $75 \mathrm{ps}$ for $T_{1}$ and $\tau \gg T_{2}^{*}$, the lower bound of $T_{2}^{*}$ can estimated to be 40 ps. Possible mechanisms for the ensemble spin dephasing $\left(T_{2}^{*}\right)$ considered in semiconductor QDs are spin dephasing in random fluctuations of the nuclear hyperfine field [26] and a spread in electron and hole g-factors [21, 27-29], both of which have been observed to act together at low temperature [30]. To evaluate the relative importance of the two, we conducted measurements of the electron spin polarization degree in a longitudinal magnetic field. If the random fluctuations of the nuclear hyperfine field introduce an effective transverse field driving spin dephasing, this can be gradually suppressed in an increasing longitudinal field [26] such that spin precession is taking place around the direction of the total field. This will lead to a dip around zero field in a polarization curve as a function of the longitudinal field strength, where the dip width should reflect the strength of the effective transverse nuclear hyperfine field. Representative experimental results are shown in figure 3, obtained at $200 \mathrm{~K}$ and $270 \mathrm{~K}$. From the absence of a zero-field polarization dip we conclude that the nuclear field fluctuations do not contribute to spin dephasing at such high temperatures. The most likely spin dephasing mechanism contributing to the Hanle shape is thus a spread in g-factors of the trion's electrons, which is expected to gain importance as the transverse external magnetic field increases [31]. As there is no reason to expect strong temperature dependence for this mechanism, we can assume $T_{2}^{*}$ to be rather insensitive to temperature. 


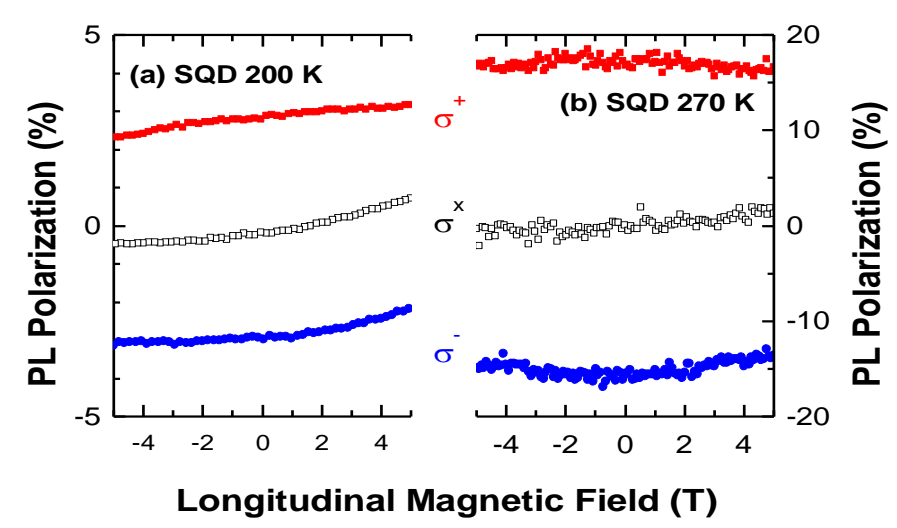

Figure 3. PL polarization as a function of a longitudinal magnetic field for the SQD sample at (a) $200 \mathrm{~K}$ and (b) $270 \mathrm{~K}$.

In contrast to the observed constant $g T_{\mathrm{s}}$, the zero-field polarization degree $P(0)$ sharply rises with increasing temperature for all samples, reaching close to $40 \%$ in the SQD sample close to RT as shown in figures $2(\mathrm{a})-(\mathrm{c}) . P(0)$ is known to be determined by

$$
P(0)=\frac{-\rho_{0}}{1+\tau / \tau_{s}}=-\beta \rho_{0} \text {. }
$$

Here $\beta=\left(1+\tau / \tau_{s}\right)^{-1}$ denotes the QD spin detection efficiency and $\rho_{0}$ is the electron spin polarization of the trions created under optical orientation (e.g. injected from the WL or GaAs barrier) at zero magnetic field before undergoing spin relaxation in the QDs. As we can exclude a significant effect of the hyperfine induced dephasing from the studies in a longitudinal magnetic field following the discussion above, and also the influence of a g-factor spread is absent in zero magnetic field, $\tau_{s}$ is mainly contributed by the longitudinal spin relaxation time $T_{1}$ in zero field.

The observed rising of $P(0)$ with increasing temperature should then be caused by an increase in spin detection efficiency $\beta$ or by an increase in spin injection efficiency leading to a larger value of $\rho_{0}$, or both. A contribution from an increasing $\beta$ is possible only if the trion decay time $\tau$ shortens with increasing temperature, assuming $T_{1}$ is temperature independent. The observed drastic thermal quenching of PL intensity is suggestive that this likely occurs [11]. As the maximum spin polarization by spin injection is $\rho_{0}=100 \%$, the trion decay time is restricted to $\tau \leq 1.2 \mathrm{~ns}$ at $220 \mathrm{~K}$ and $\tau \leq 130 \mathrm{ps}$ at $290 \mathrm{~K}$, obtained by using the experimentally measured values of $P(0)=6 \%$ at $220 \mathrm{~K}$ and $P(0)=40 \%$ at $290 \mathrm{~K}$ (figure 2(a)) from the SQD sample and assuming $T_{1} \approx 75 \mathrm{ps}$ [25]. On the other hand, $\tau$ must be much larger than the $T_{\mathrm{s}}$ value determined from the Hanle measurements as discussed above. Hence, we arrive at the conclusion that the maximum extent of the trion lifetime shortening is restricted to about one order of magnitude between $220 \mathrm{~K}$ and $290 \mathrm{~K}$, in good agreement with earlier results $[32,33]$. The PL intensity, however, has been found to decrease by two orders of magnitude over the same temperature range [11], which is more than what is expected from the shortening of the trion lifetime alone. This discrepancy can be accounted for if the number of photogenerated carriers injected from the WL has dropped over this temperature range, contributing to the decrease in the PL intensity of the monitored QD trions. This corresponds to a reduction of carrier lifetime in the WL, likely due to thermally activated non-radiative carrier recombination via defects, which in turn will restrict the extent of spin relaxation in the WL. The resulting higher electron spin polarization in the WL leads to an increase in spin polarization of the injected electrons $\rho_{0}$ and thus contributes to the observed rising of the QD ground state polarization degree. Therefore, the observed rising in $P(0)$ with increasing temperature should be induced by an increase in both spin injection efficiency from the WL and spin detection efficiency of the QDs.

In addition, we also investigated the electron spin lifetime of the positive trions in the QDs under various excitation densities. It is found that $g T_{\mathrm{s}}$ is nearly independent of optical excitation power, as shown in figure 4 . This finding is quite understandable in the framework that the largest contribution 
to the Hanle width is the spin dephasing of the QD ensemble, as no change of $T_{2}^{*}$ with excitation power is expected. The accompanying change in PL polarization could then be explained by an increasing contribution from unpolarized biexciton emission, originating from filling of a second electron with an opposite spin orientation. For completeness, we also studied the spin lifetime of the excited states of the QDs. Representative results are shown in figure 5. A moderate broadening in the width of the Hanle curves is observed for the excited states, corresponding to a shortening of their scaled spin lifetime. In view of the observed accompanying increase in PL polarization, it is tempting to suggest that this could be caused by a shorter lifetime of the relevant excitons. However, earlier results [23] have shown that the exciton decay times of the excited states are in fact very similar to that of the exciton ground state at high temperatures, due to efficient thermalization between these states. This rules out a shortening of exciton decay times as being the reason for the observed broadening of the Hanle curves, and leaves a slightly shortened $T_{1}$ and $T_{2}^{*}$ as likely causes. The observed higher spin polarization of the exciton excited states, on the other hand, is probably due to a lower probability of being filled with spin paired-off electrons in the excited states as compared with the ground state.

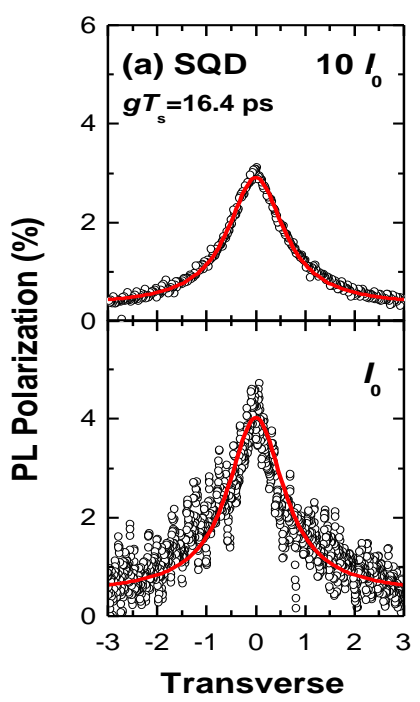

Magnetic Field (T)

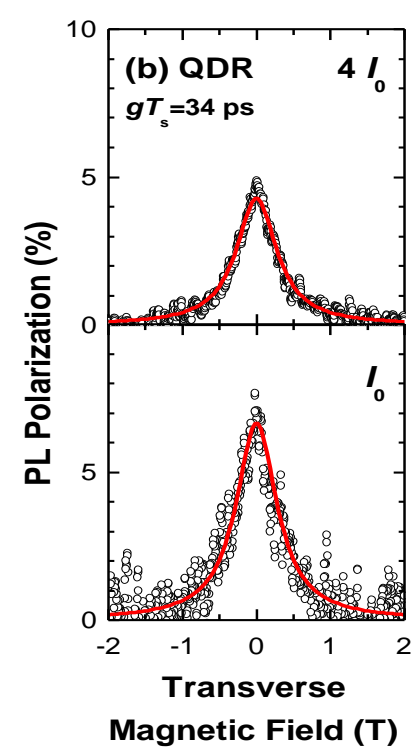

Magnetic Field (T)

Figure 4. Hanle curves from the SQD (a) and QDR (b) sample under two different excitation powers, obtained at $160 \mathrm{~K}$ under optical orientation in the WL.

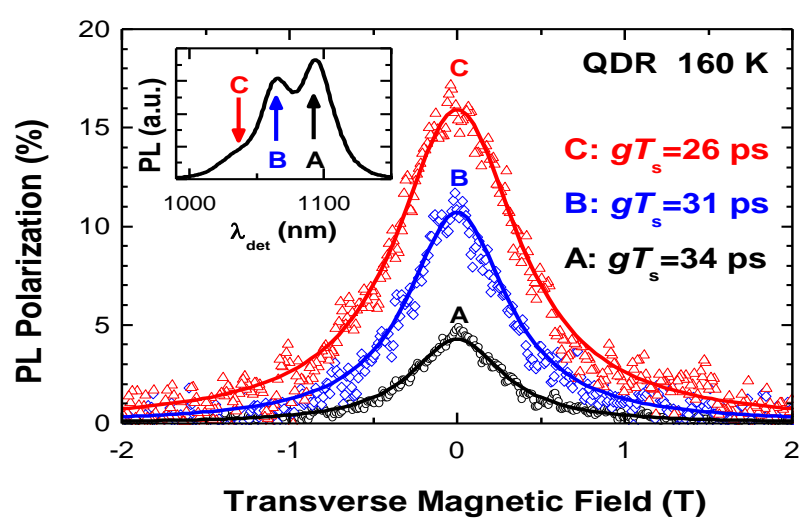

Figure 5. Hanle curves from the QDR sample under optical orientation in the WL, obtained at 160 $\mathrm{K}$ by detecting at the ground and excited states of the QDs as indicated by the arrows in the PL spectrum shown in the inset. 


\section{Summary}

From studies of the Hanle effect, we observed a constant spin lifetime in the investigated InAs/GaAs QDs over a wide range of temperatures close to RT. The observation can be attributed to temperature-insensitive spin-flip relaxation and spin dephasing of the monitored QD trions, with the latter being most likely related to a spread in the electron g-factor of the QD ensemble. The observed rise in QD spin polarization degree with increasing temperature is argued to be contributed by an increase in both spin injection efficiency from the WL/barrier, promoted by thermal activation of nonradiative carrier recombination in the spin injection layers (i.e. WL and barrier), and a moderate increase in spin detection efficiency of the QD trion ground state. 


\section{References}

[1] Burkard G, Engel H-A and Loss D 2000 Fortschr. Phys. 48965

[2] Loss D and DiVincenzo D P 1998 Phys. Rev. A 57120

[3] C. Simon et al 2010 Europ. Phys. J. D 581

[4] Heiss D, Kroutvar M, Finley J and Abstreiter G 2005 Solid State Commun. 135591

[5] Chamarro M, Bernardot F and Testelin C 2007 J. Phys.: Cond. Matt. 19445007

[6] Bracker A, Gammon D and Korenev V 2008 Semicond. Sci. Technol. 23114004

[7] Hanson R and Awschalom D 2008 Nature 4531043

[8] Kusrayev Y 2008 Semicond. Sci. Technol. 23114013

[9] Coish W and Baugh J 2009 Phys. Status Solidi B 2462203

[10] Ramsay A 2010 Semicond. Sci. Technol. 25103001

[11] Beyer J, Buyanova I A, Suraprapapich S, Tu C W and Chen W M 2011 Appl. Phys. Lett. 98 203110

[12] Dyakonov M I 2008 Spin Physics in Semiconductors (Berlin Heidelberg: Springer)

[13] Bracker A S, Stinaff E A, Gammon D, Ware M E, Tischler J G, Shabaev A, Efros A L, Park D, Gershoni D, Korenev V L and Merkulov I A 2005 Phys. Rev. Lett. 94047402

[14]Epstein R J, Fuchs D T, Schoenfeld W V, Petroff P M and Awschalom D D 2001 Appl. Phys. Lett. 78733

[15] Masumoto Y, Oguchi S, Pal B and Ikezawa M 2006 Phys. Rev. B 74205332

[16] Shabaev A, Stinaff E A, Bracker A S, Gammon D, Efros A L, Korenev V L and Merkulov I 2009 Phys. Rev. B 79035322

[17] Pal B and Masumoto Y 2009 Phys. Rev. B 80125334

[18] Suraprapapich S, Panyakeow S and Tu C W 2007 Appl. Phys. Lett. 90183112

[19] Suraprapapich S, Shen Y M, Odnoblyudov V A, Fainman Y, Panyakeow S and Tu C W 2007 J. Cryst. Growth 301-302 735

[20] Eble B, Testelin C, Desfonds P, Bernardot F, Balocchi A, Amand T, Miard A, Lemaittre A, Marie $\mathrm{X}$ and Chamarro M 2009 Phys. Rev. Lett. 102146601

[21] Yugova I A, Greilich A, Zhukov E A, Yakovlev D R, Bayer M, Reuter D and Wieck A D 2007 Phys. Rev. B 75195325

[22] Melliti A, Maaref M, Hassen F, Hjiri M, Maaref H, Tignon J and Sermage B 2003 Solid State Comm. 128213

[23] Gurioli M, Vinattieri A, Zamfirescu M, Colocci M, Sanguinetti S and Nötzel R 2006 Phys. Rev. B 73085302

[24] Harbord E, Spencer P, Clarke E and Murray R 2009 Phys. Rev. B 80195312

[25] Marcinkevičius S, Siegert J and Zhao Q X 2006 J. Appl. Phys. 100054310

[26] Braun P F et al 2005 Phys. Rev. Lett. 94116601

[27] Gupta J A, Awschalom D D, Peng X and Alivisatos A P 1999 Phys. Rev. B 59 R10421

[28] Kennedy T A, Bracker A S, Carter S G, Economou S E, Gammon D, Whitaker J, Hasan Z U, Craig A E and Hemmer P R 2008 Proc. SPIE 6903 69030Q

[29] Andreev S V, Namozov B R, Koudinov A V, Kusrayev Y G and Furdyna J K 2009 Phys. Rev. B 80113301

[30] Lombez L, Braun P-F, Marie X, Renucci P, Urbaszek B, Amand T, Krebs O and Voisin P 2007 Phys. Rev. B 75195314

[31] At $1 \mathrm{~T}$, an estimated spread of electron g-value is $\Delta g=\hbar / \mu_{B} B T_{2}^{*}=0.28$, which is $56 \%$ of the mean $g=0.5$. Such large fluctuation degree was earlier observed for the hole in a negative trion, as the in-plane hole g-value is extremely sensitive to structural distortion that admixes the hh and lh state. As a result, the Hanle curve was described by a non-Lorentzian lineshape that could be regarded as a signature of a hole-related spin depolarization, see Ref. [29]. A similar large fluctuation in $\mathrm{g}$ value of electrons is, however, not expected. Still rather large spreads in electron g-values of $\Delta g=0.1$ have been reported, see Ref. [28]. At present the exact mechanism for the estimated large fluctuation in the $\mathrm{g}$ value of the positive trion electrons is unknown, which requires further studies. 
[32] Heitz R, Mukhametzhanov I, Madhukar A, Hoffmann A and Bimberg D 1999 J. Electron. Mater. 28520

[33] Lee U H, Yim J S, Lee D, Lee H G, Noh S K, Leem J Y and Lee H J 2000 J. Korean Phys. Soc. 37593 